\title{
Caracterización litológica y mineralógica de los materiales permo-triásicos de Castellón utilizados como materia prima cerámica: la arcilla de Moró
}

\author{
J.D. MARTíN-MARTíN*1, T. SANFELIU², D. GÓMEZ-GRAS ${ }^{3}$, C. DE LA FUENTE", M. JORDÁN ${ }^{5}$ \\ 1 Department of Earth Sciences, Uppsala University, SE-75236 Uppsala, Sweden \\ 2 Departament de Ciències Experimentals, Universitat Jaume I, 12080 Castelló de la Plana, Spain \\ 3 Departament de Geologia, Universitat Autònoma de Barcelona, 08193 Bellaterra, Spain \\ 4 Departament de Cristal·lografia, Mineralogia i Dipòsits Minerals, Universitat de Barcelona, 08028, Barcelona, Spain \\ 5 Departamento de Agroquímica y Medio Ambiente, Universidad Miguel Hernández, 03202 Elche, Spain
}

\begin{abstract}
Las lutitas de edad Pérmica que se utilizan como materia prima en la industria azulejera de Castellón se conocen en el sector cerámico como arcilla de Moró. En el presente trabajo se describen las características litológicas y composicionales de estas lutitas con el objetivo de establecer las variaciones en composición a lo largo de la serie estratigráfica tipo. Los resultados muestran una composición mineralógica de la roca total muy uniforme, formada esencialmente por cuarzo, filosilicatos y hematites. Ocasionalmente y en proporciones por debajo del 5\% aparece también feldespato potásito, dolomita y calcita. La asociación de minerales de la arcilla está formada por illita \pm caolinita \pm clorita \pm interestratificado illita-esmectita \pm interestratificado clorita-esmectita y muestra un patrón de distribución sedimentario. Los resultados obtenidos ponen de manifiesto la importancia del análisis mineralógico en la caracterización de las materias primas y, por tanto, en el control de sus propiedades cerámicas y de los procesos productivos relacionados.
\end{abstract}

Palabras clave: arcilla, mineralogía, materia prima, industria cerámica, Permo-Triásico

Lithology and mineralogy of the Permo-Triassic raw materials used in the tile industry of Castellón (Spain): the Moró clay.

The aim of this work is to establish the mineralogical (XRD) composition of the Permo-Triassic claystone known as Moró clay. Particular emphasis is placed on the compositional variations along the litostratigraphic section. Bulk rock XRD analysis shows a homogeneous composition dominated by quartz, phyllosilicates and hematite. Minor components $(<5 \%)$ include potassium feldspar, dolomite and calcite. The clay minerals assemblage is formed by illite \pm kaolin \pm chlorite \pm mixed-layer illite-smectite \pm mixed-layer chlorite-smectite and reveals a sedimentary distribution pattern. The study of the lithological section allows the identification and valuation of changes in the mineralogical characteristics of the raw material. Results provide evidence for the importance of mineralogical analysis in the control of raw materials and hence their use in industrial processes.

Keywords: clay, raw material, mineralogy, tile industry, Permo-Triassic

\section{INTRODUCCIÓN}

Una característica fundamental de los depósitos sedimentarios arcillosos que abastecen de materia primas a la industria azulejera de Castellón es su heterogeneidad mineralógica $(1,2,3)$. A nivel productivo este aspecto se traduce en una variabilidad importante de las propiedades cerámicas del producto, tanto en crudo como en cocido (1, $2,3)$. Tradicionalmente este inconveniente se ha minimizado mediante la utilización de sistemas de homogeneización en las propias explotaciones mineras, en las atomizadoras o bien en las empresas productoras $(1,2)$ (Fig. 1). Los problemas derivados de la materia prima pueden reducirse a partir del estudio de su composición mineralógica y de los parámetros geológicos que influyen en su distribución, como paso previo a la investigación de los productos cerámicos acabados $(4,5)$. El estudio mineralógico del yacimiento facilita, además, el control minero de las materias primas desde la fase de prospección geológica (5). El objetivo de este trabajo es caracterizar litológica y mineralógicamente la formación geológica que se explota como arcilla de Moró, a fin de estudiar aquellos aspectos que puedan tener influencia en su explotación como materia prima cerámica. Los datos que se presentan forman parte de un trabajo con finalidad científica más amplia sobre las arcillas permo-triásicas de la provincia de Castellón que constituye la Tesis Doctoral del primer firmante (6).

Con el nombre de arcilla de Moró se conoce comercialmente una de las materias primas más utilizadas por el sector cerámico castellonense $(7,8)$. La denominación es originaria del municipio de Sant Joan de Moró (Castellón) donde se explota desde la década de los 70, inicialmente para ladrillería y más tarde para pavimento y revestimiento cerámico (Fig. 2). La proximidad de las explotaciones a los centros productores ha favorecido su incorporación a la formulación de pastas 


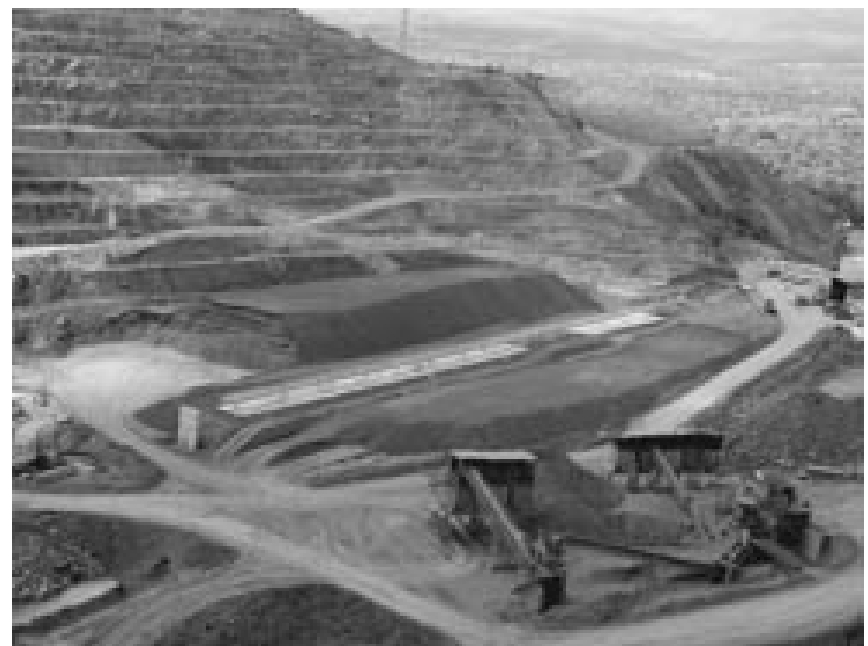

Fig. 1- Imagen que muestra las pilas de homogenización (centro) en una explotación de arcillas. rojas junto con otras materias primas clásicas como las arcillas de Villar del Arzobispo (Valencia) o de Galve (Teruel), y con cierta ventaja sobre nuevos yacimientos como los de Morella (Castellón) (9). Desde el punto de vista cerámico la arcilla de Moró se clasifica como una materia prima de bajo contenido en carbonatos $(<5 \%)$, utilizada fundamentalmente para la fabricación de gres esmaltado de pasta roja por prensado (3). Entre las características más importantes, comparada con otras materias primas para formulaciones de pasta roja, destacan su relativa alta compacidad y baja plasticidad (10). Geológicamente se trata de lutitas compactas de color rojo y edad Pérmico superior (11).

Aunque las explotaciones de arcilla de Moró se concentran entre los municipios de Sant Joan de Moró y Vilafamés, sus características geológicas, tanto estratigráficas como mineralógicas, pueden hacerse extensivas a toda la zona conocida geológicamente como Desert de les Palmes (6) (Fig. 3). La zona se caracteriza por la presencia de un conjunto de fallas normales de dirección NE-SO, y vergencia principal hasta el NO, que permite el afloramiento del zócalo hercínico, el Pérmico y el Triásico suprayacente, y la cobertera jurásica y cretácica de potencia muy reducida (12). El zócalo hercínico está formado por pizarras y grauvacas de edad Carbonífero

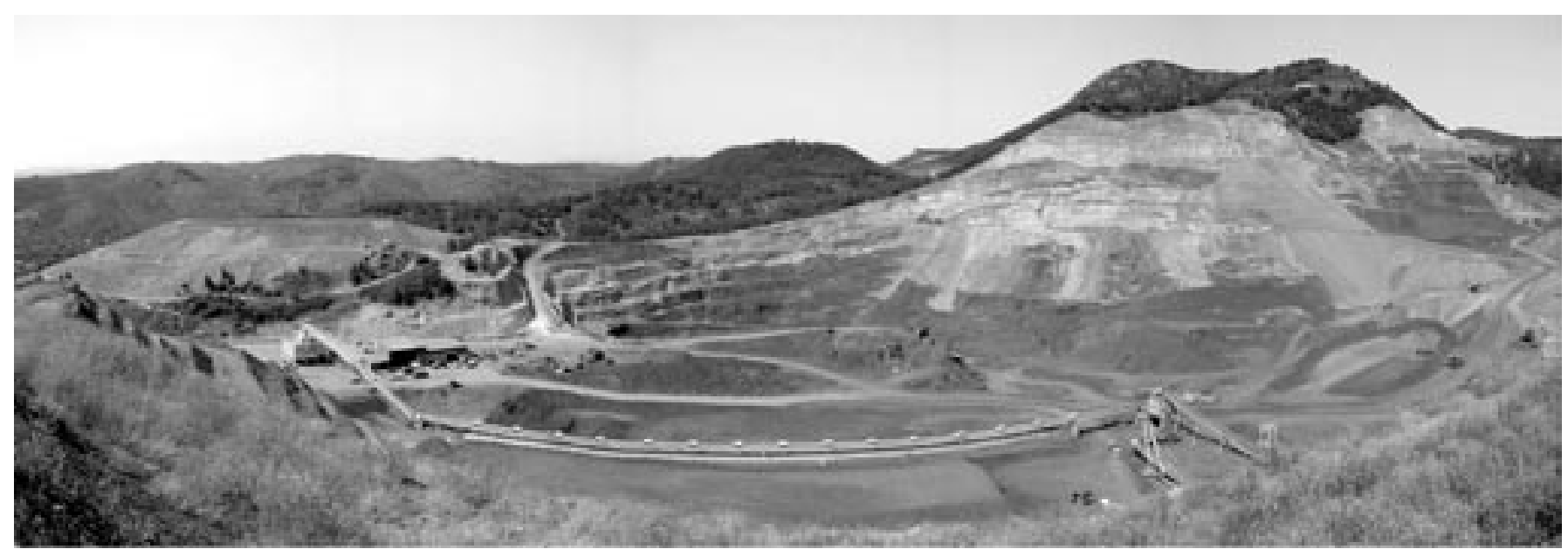

Fig. 2- Aspecto de la explotación de arcilla de Moró de WBB España en Sant Joan de Moró (Castellón).

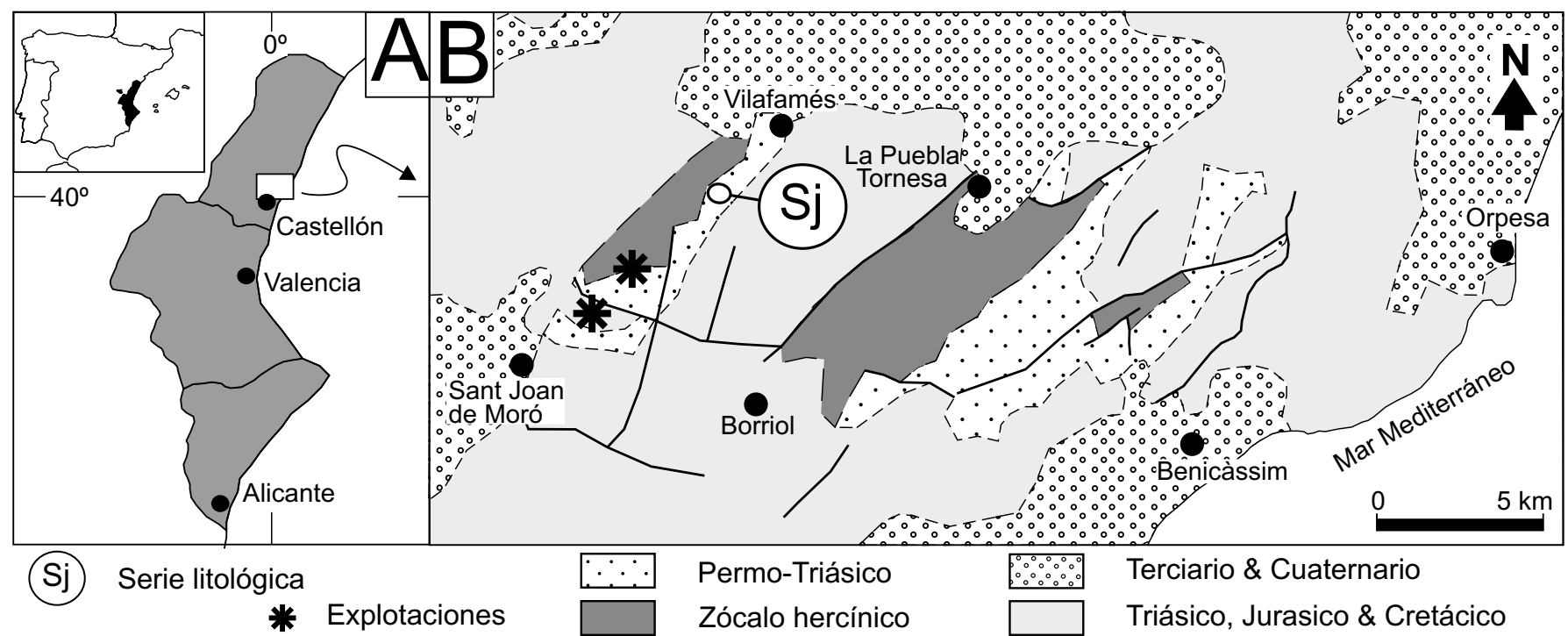

Fig. 3- (A) Situación de la zona de estudio en el contexto de la Comunidad Valenciana. (B) Mapa geológico simplificado del Desert de les Palmes. 
(13). Discordantemente sobre los anteriores se sitúa el Pérmico y el Triásico continentales (facies Saxoniense y Buntsadstein), constituidos por una sucesión de conglomerados, areniscas y lutitas de color rojo (red beds). Esta sucesión (en adelante Permo-Triásico) constituye un conjunto de sedimentos de color rojo que puede dividirse en tres unidades litológicas. La unidad inferior es de edad Pérmico Superior (Thuringiense) y se depositó en un ambiente fluvial meandriforme $(14,15)$. La unidad intermedia es de edad Triásico inferior (Escitiense) y es característica de un régimen aluvial trenzado $(14,15)$. La unidad superior es de edad Triásico medio (Anisiense) y se depositó también en un ambiente fluvial meandriforme (14, 15). Estas tres unidades son equivalentes a las descritas en $(15,16)$ en la zona del Desert de les Palmes o a las definidas con rango de formación en (14) para toda la Cordillera Ibérica oriental.

\section{METODOLOGÍA}

Se ha realizado la caracterización litológica y estratigráfica de la serie permo-triásica local así como el muestreo sistemático de los tramos lutíticos para su análisis mineralógico. El muestreo abarca tanto los tramos más potentes, y por ello potencialmente explotables como materia prima arcillosa, como aquellos de espesor centimétrico y sin importancia desde ese punto de vista.

El análisis mineralógico de las lutitas se ha realizado mediante difracción de rayos $X$ (DRX) en un equipo Phillips PW $1710(\mathrm{Cu}-\mathrm{K} \alpha)$. Se ha determinado la mineralogía de la roca total (agregado desorientado) y de la fracción arcilla (agregados orientados sin tratar, saturado en atmósfera de etilién-glicol durante 12 horas y tras calentamiento en horno a $490^{\circ} \mathrm{C}$ durante 4 horas). La desagregación de la muestra previa a su análisis se ha realizado mediante agitación mecánica por via húmeda a fin de no alterar su granulometría original. La preparación del agregado orientado incluye la extracción de la fracción arcilla $(<2 \mu \mathrm{m})$ mediante decantación, la eliminación de carbonatos mediante ataque ácido y centrifugación, y el depósito de una parte de la suspensión resultante sobre una placa de vidrio. El porcentaje de cada fase mineral se ha estimado de forma semicuantitativa a partir del área de la reflexión característica de cada especie multiplicada por un factor de difracción relativo a la naturaleza del mineral en cuestión y normalizado al 100\% (17).

\section{RESULTADOS}

\subsection{Descripción de la serie permo-triásica}

La columna litológica analizada aflora en la vertiente occidental de la sierra de Vilafamés y presenta una potencia total de $423 \mathrm{~m}$ distribuidos en tres unidades litológicas (Fig. 3 y 4). La unidad inferior alcanza una potencia de $237 \mathrm{~m}$ aunque, probablemente, este valor sea mayor debido a la presencia de numerosos tramos cubiertos (Fig. 4). La unidad inferior está formada por lutitas (arcillitas y limolitas) rojas y compactas con frecuentes intercalaciones de areniscas del mismo color. La base de esta unidad es fácilmente reconocible por la presencia de un conglomerado de cantos de cuarzo subredondeados que sobrepasa los $10 \mathrm{~m}$ de potencia. Las lutitas forman tramos que pueden superar los $20 \mathrm{~m}$ de potencia y dominan ampliamente en la primera mitad de la unidad. Un detalle importante

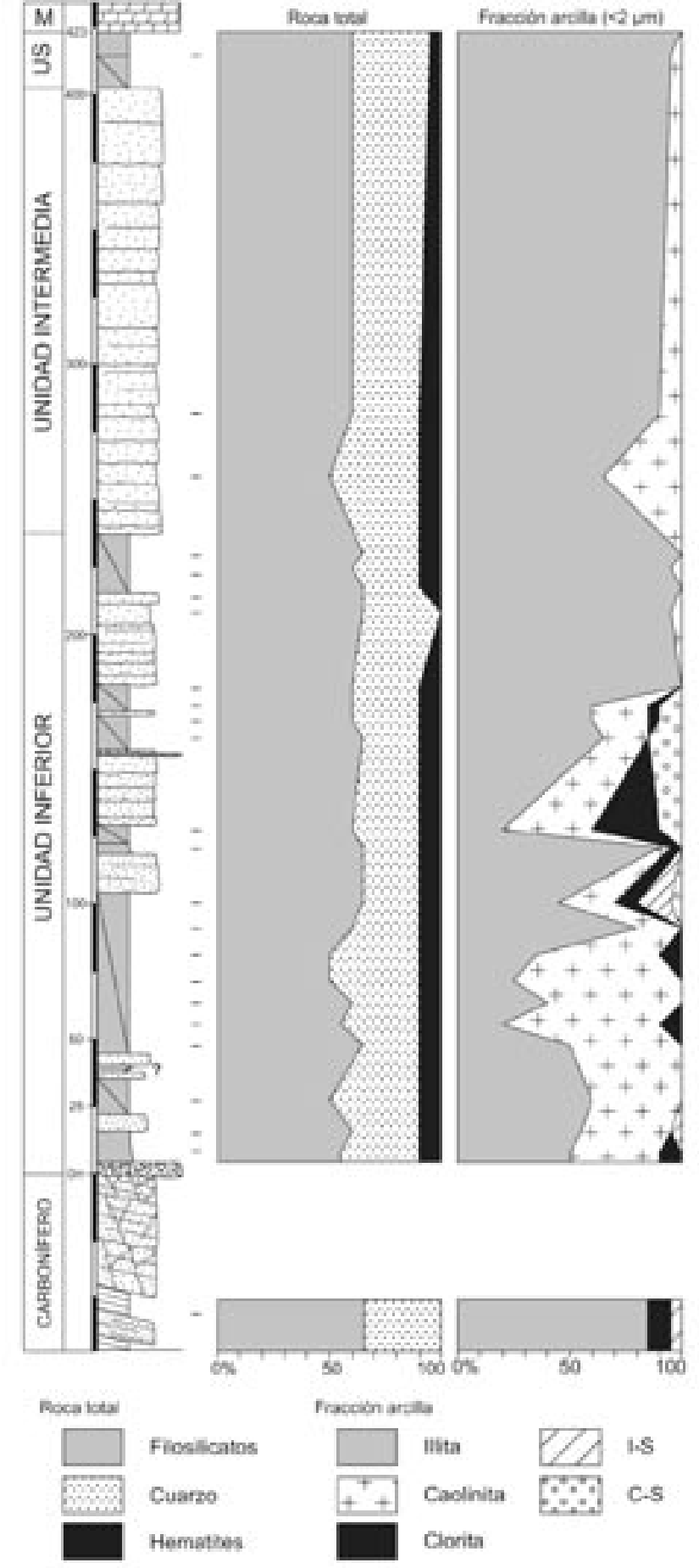

Fig. 4- Columna estratigráfica de Sant Joan de Moró junto a la evolución mineralógica vertical de la roca total y de la fracción arcilla. La localización de las muestras de lutitas está marcada con un símbolo (-). (US) Unidad superior; (M) Muschelkalk.

en relación a estos tramos es la presencia de fragmentos milimétricos de rocas metamórficas (pizarras), con forma de lenteja, que denota un origen detrítico a partir del zócalo hercínico infrayacente. Estas partículas aparecen entre la matriz arcillosa y, ocasionalmente, se concentran formando estratos microconglomeráticos. No se ha observado la existencia de 
paleosuelos aunque en ocasiones se han reconocido nódulos de caliche de tamaño milimétrico. En muestra de mano las lutitas muestran un brillo muy característico debido a su carácter micáceo.

Las areniscas se ordenan en estratos métricos de grano fino, con estratificación cruzada y paralela. El color suele ser rojo y menos frecuentemente ocre o gris. La base es neta y el techo suele ser transicional a los niveles de lutitas. En la segunda mitad de la unidad inferior, los estratos de areniscas se amalgaman mediante superficies de erosión para formar conjuntos que superan los $15 \mathrm{~m}$ de potencia. Entre los tramos de lutitas también aparecen estratos de arenisca de poco espesor y tamaño de grano muy fino, aunque no se han representado en la columna litológica por problemas de escala. La unidad intermedia presenta una potencia aproximada de $165,5 \mathrm{~m}$ y está formada exclusivamente por areniscas micáceas de color rojo o naranja. El conjunto es muy resistente a la erosión y dibuja un resalte característico en el paisaje de la sierra de Vilafamés y, en general, en toda la zona del Desert de les Palmes. Las areniscas tienen un tamaño de grano medio y se organizan en estratos de orden métrico con estratificación cruzada, a mediana y gran escala, y ocasionalmente en surco. Los estratos de lutitas son muy escasos y apenas alcanzan 10 o $20 \mathrm{~cm}$ de espesor. La unidad superior presenta $21 \mathrm{~m}$ de potencia y está formada por lutitas rojas con intercalaciones muy finas de areniscas. En los últimos metros aparecen arcillas, limos y margas de color rojo, verde y ocre, muy deformadas debido al carácter mecánico del contacto con las calizas suprayacentes de las facies Muschelkalk.

\subsection{Composición mineralógica}

La composición mineralógica de la roca total es muy homogénea a lo largo de toda la serie permo-triásica y está formada fundamentalmente por cuarzo, filosilicatos y hematites (Fig. 4). También se ha identificado, aunque no en todas las muestras y siempre en proporciones muy bajas, la presencia de feldespato potásico, calcita y dolomita. Todos estos minerales han sido considerados únicamente como existentes $(<5 \%)$.

Las lutitas de la unidad inferior presentan una fracción arcilla formada por illita y caolinita acompañadas de porcentajes menores, cuando existen, de clorita y minerales interestratificados I-S y C-S (Fig. 4). La denominación de illita engloba al grupo de filosilicatos dioctédricos 2:1 en el que se incluyen diferentes tipos de minerales micáceos como la illita s.s. y las moscovitas detríticas que hayan podido entrar a formar parte de la fracción arcilla durante el proceso de desagregación. En la distribución de base a techo de la unidad se observa un aumento progresivo de la illita, hasta alcanzar el $100 \%$ de la muestra, y una disminución de la caolinita y del resto de mineralogías. Tanto la clorita como los minerales interestratificados son más frecuentes en la primera mitad de la unidad inferior, raramente superan el $10 \%$ de la muestra y desaparecen por completo antes de llegar a la unidad intermedia. A partir de la unidad intermedia la illita y la caolinita pasan a ser los únicos minerales presentes en la fracción arcilla de la lutitas. Así, en la unidad superior la illita es mayoritaria y la caolinita aparece en porcentajes poco significativos.

\section{DISCUSIÓN}

Desde el punto de vista estrictamente litológico la unidad inferior (Pérmico Superior) es la más adecuada para la explotación de las lutitas como materia prima cerámica (11). La litología dominantemente areniscosa de la unidad intermedia y la poca potencia de la unidad superior limitan su posible interés cerámico (11). La sucesión de estratos de lutitas y areniscas que caracteriza la mayor parte de la unidad inferior es representativa de un ambiente de llanura de inundación surcada por canales entrelazados $(14,15)$. Las areniscas representan el relleno de los canales fluviales mientras que las lutitas, de granulometría mucho más fina, representan el material de desbordamiento. Estos tramos lutíticos son más frecuentes en la primera mitad de la unidad inferior y corresponden a los materiales que se explotan como materia prima cerámica.

Los resultados promediados por unidades litológicas permiten estimar la composición mineralógica de la unidad inferior y, por tanto, de la arcilla de Moró (Tabla 1). La composición mineralógica resultante es similar a la descrita por otros autores a partir de muestras puntuales o previamente homogeneizadas $(1,3,10,18)$. En el caso de la roca total, la composición denota una relación cuarzo/filosilicatos aproximada de $32 / 59 \%$. La proporción relativa de estos dos minerales está directamente relacionada con la granulometría de la muestra. Los filosilicatos dominan en las muestras de granulometría más fina (arcillitas) mientras que las muestras con mayor contenido en cuarzo pueden considerarse limolitas o incluso areniscas de tamaño de grano muy fino. A nivel de explotación prácticamente no existe distinción entre unas y otras, y todas entran a formar parte de la materia prima homogeneizada. Este detalle pone de relieve dos de las principales características cerámicas de la arcilla de Moró. Por un lado el contenido en cuarzo suele ser elevado y, por otro, presenta una importante proporción de partículas de elevado tamaño $(1,3,18)$. En el sector cerámico este detalle se valora mediante el parámetro conocido como rechazo, equivalente al porcentaje en peso de partículas de tamaño superior a 63 $\mu \mathrm{m}$. Otro aspecto que contribuye a la elevada proporción de partículas gruesas de la arcilla de Moró son los fragmentos milimétricos de pizarra que aparecen entre la matriz arcillosa (18). Estas partículas están formadas fundamentalmente por cuarzo, illita y clorita, y provienen directamente de la erosión

TABLA I. COMPOSICIÓN MINERALÓGICA PROMEDIADA POR UNIDADES LITOLÓGICAS DE LA COLUMNA ESTRATIGRÁFICA DE SANT JOAN DE MORÓ (\%).

\begin{tabular}{|c|c|c|c|c|c|c|c|c|c|c|c|}
\hline & & \multicolumn{5}{|c|}{ Roca total } & \multicolumn{5}{|c|}{ Fracción arcilla } \\
\hline Litología & Unidad & Q & Fk & Fil & $\mathrm{Ca}$ & $\mathrm{Hm}$ & I & $\mathrm{K}$ & C & I-S & C-S \\
\hline & Us (1) & 35 & $x$ & 60 & - & 5 & 95 & 5 & - & - & - \\
\hline Lutitas & Um (2) & 35 & $x$ & 55 & $x$ & 10 & 77 & 23 & - & - & - \\
\hline & $\mathrm{Ui}(20)$ & 32 & $x$ & 59 & $x$ & 9 & 62 & 30 & 4 & 1 & 3 \\
\hline
\end{tabular}

Us: unidad superior; Um: unidad intermedia; Ui: unidad inferior. Entre paréntesis se indica el número de muestras. Q: Cuarzo; Fk: Feldespato potásico; Fil: Filosilicatos; Ca: Carbonatos; Hm: Hematites; I: Illita; K: Caolinita; C: Clorita; I-S: Interestratificado illita-esmectita; C-S: Interestratificado clorita-esmectita, x: Existente (sin cuantificar). 
del zócalo carbonífero infrayacente tal como demuestra la mineralogía de las pizarras (Fig. 4). Tanto el cuarzo como la pizarra se comportan como desgrasantes, aumentan la compactación natural de la materia prima y regulan la deformación de la pieza durante la cocción $(1,3,18)$.

El color rojo característico de toda la serie permo-triásica es debido a la presencia de hematites aunque excepcionalmente aparecen estratos de color gris, intercalados entre las capas rojas, cuyo contenido en este mineral es muy inferior. El elevado contenido en hematites condiciona que la arcilla de Moró, igual que otras materias primas de la misma tipología, se utilice fundamentalmente en la fabricación de soportes de pasta roja $(1,3)$.

El carbonato más abundante a lo largo de toda la serie es la dolomita y su origen está relacionado, probablemente, con la existencia de cemento dolomítico en los estratos de granulometría más gruesa (areniscas) (15). La proporción de calcita es muy baja en todas las muestras estudiadas aunque valores sensiblemente superiores $(>5 \%)$, de gran importancia por su efecto nocivo en las piezas cerámicas durante el proceso de cocción, han sido descritos por varios autores $(1,2,3)$. La variabilidad en la proporción de calcita se relaciona con la existencia de nódulos de caliche cuya formación tiene su origen en los procesos pedogenéticos que se desarrollan en ambientes fluviales como los que caracterizan a la unidad inferior. Durante el trabajo de campo se ha reconocido la existencia de nódulos de caliche en algunos estratos de areniscas situados en la base de la serie y, por tanto, su existencia puntual es muy probable en las lutitas. El bajo contenido en carbonatos $(<5 \%)$ es otra de las principales características fundamentales de la arcilla de Moró y es un requisito fundamental para su uso en la fabricación del pavimento gresificado (3).

La asociación de minerales de la arcilla característica de la unidad inferior, y de la arcilla de Moró, está formada por illita \pm caolinita \pm clorita \pm I-S \pm C-S (Tabla 1 ). Esta asociación y su distribución a lo largo de la unidad inferior reflejan un origen fundamentalmente detrítico del conjunto (6). El origen de la caolinita se relaciona básicamente con la erosión de perfiles de meteorización ricos en este mineral que se desarrollaron sobre el zócalo hercínico del área fuente con anterioridad a la sedimentación de la serie permo-triásica $(19,20)$. La illita constituye el principal componente detrítico de toda la serie permo-triásica y proviene de la erosión de las rocas metamórficas de bajo grado, y en menor media graníticas, aflorantes en el área fuente $(21,20)$. En los diagramas de RX de las muestras correspondientes a la unidad inferior aparecen picos de difracción a $\sim 10 \AA$ sensiblemente más anchos que en las dos unidades superiores, lo que sugiere la existencia de illitas degradadas en la base de la serie. Esta evolución vertical indica una mayor erosión del zócalo metamórfico sin meteorizar durante la sedimentación de las unidades triásicas. La clorita y los minerales interestratificados C-S provienen igualmente de la erosión de las rocas metamórficas que forman el zócalo hercínico infrayacente $(22,20)$. La presencia de clorita en las lutitas de la unidad inferior es una característica importante de estos materiales puesto que aumenta la fundencia de la materia prima cerámica (3).

La presencia de caolinita, así como la de interestratificados I-S, puede tener su origen en la alteración de micas y fragmentos de rocas durante la diagenesis temprana tal como pone de manifiesto (23), aunque su contribución en el caso de la unidad inferior no se considera muy importante teniendo en cuenta la limitada permeabilidad asociada a las lutitas. Un aumento del contenido de caolinita relacionado con la diagenesis temprana es mucho más factible en los estratos de lutitas de orden centimétrico característicos de la unidad intermedia y situados entre areniscas de permeabilidad mucho más elevada.

\section{CONCLUSIONES}

Las lutitas que se explotan como materia prima cerámica en la zona del Desert de les Palmes (Castellón) constituyen la unidad inferior de la serie permo-triásica y su edad es Pérmico superior (Thuringiense). Estos materiales muestran las heterogeneidades típicas de los sedimentos fluviales (carbonatos y relación cuarzo/filosilicatos), cuya existencia y proporción puede estimarse a partir del análisis sistemático de la sucesión litológica característica de la zona.

La asociación de minerales de la arcilla que caracteriza a la unidad inferior (Pérmico) es típica de las series permo-triásicas de margen de cuenca. La distribución de los minerales de la arcilla a lo largo de la columna litológica muestra un patrón de distribución sedimentario y, por tanto, es predecible en el contexto del Desert de les Palmes. El patrón de distribución descrito es similar al que presentan otras series permotriásicas de la provincia de Castellón (sierras de Espadá y Calderona) (6). La caolinita se concentra en la base de la serie y su proporción disminuye considerablemente hacia el techo de la unidad inferior a favor de la illita. La proporción más elevada de clorita aparece en la primera mitad de la unidad inferior.

El estudio mineralógico realizado permite la caracterización detallada de la materia prima (arcilla de Moró) a priori o durante el proceso de explotación. Por otro lado, los resultados obtenidos facilitan la optimización de los sistemas de homogenización y, por tanto, el control de las características cerámicas de la materia prima.

\section{AGRADECIMIENTOS}

Los autores agradecen a la empresa WBB España S.A. su colaboración en la realización de este trabajo. Juan Diego Martín agradece a la Generalitat Valenciana la concesión de la beca post-doctoral de Formación de Personal Investigador (CTBPD/2004/069). Este trabajo ha sido parcialmente financiado por el Ministerio de Educación y Ciencia (Proyectos BTE2001-0568 y CGL2005-07445-C03-01/BTE).

\section{BIBLIOGRAFÍA}

1. García-Ten, Características técnicas y problemática actual del consumo de arcillas decocción roja, en I Jornadas sobre materias primas de la industria cerámica (Conferencias), Castellón, 7 y 8 de febrero. Asociación Española de Técnicos Cerámicos (ATC), Febrero 1996.

2. J.C. Santiago, Evolución y problemática de los recursos y reservas de materias primas en la minería a cielo abierto, en I Jornadas sobre materias primas de la industria cerámica (Conferencias), Castellón, 7 y 8 de febrero. Asociación Española de Técnicos Cerámicos (ATC), Febrero 1996.

3. A. Barba, V. Beltrán, C. Feliu, J. García, F. Ginés, E. Sanchez, V. Sanz. Materias primas para la fabricación de soportes de baldosas cerámicas, Instituto de Tecnología Cerámica - AICE, Castellón (España) 1997.

4. A. García-Verduch, Algunos aspectos de la investigación cerámica actual». Bol. Soc. Esp. Ceram. V., 12, 5, 279-288 (1973).

5. C. De la Fuente, A. Boix, T. Sanfeliu. «La investigación mineralógica en las materias primas cerámicas. Técnica Cerámica, 207 660-670 (1992).

6. J.D. Martín-Martín, Los minerales de la arcilla del Permo-Triásico de la Cordillera Ibérica oriental: procedencia y evolución diagenética, Tesis Doctoral. Universitat Jaume I (2004). 
7. E. Criado, E. Sánchez, M. Regueiro, La industria cerámica española, ¿ante un cambio de ciclo?, Bol. Soc. Esp. Ceram. V., 43, 1, 85-101 (2004).

8. E. Sánchez, J. García-Tem, M. Regueiro. Materias para la industria cerámica española. Situación actual y perpectivas. Bol. Soc. Esp. Ceram. V., 45, 1, 1-12 (2006).

9. E. Sánchez, J. García-Ten, P. Quereda, V. Beltrán, Arcilla de Morella. Nueva materia prima para la fabricación de baldosas cerámicas de pasta roja, Técnica Cerámica, 298, 1386-1396 (2001).

10. Bastida, Petrografía y mineralogía de arcillas en relación con procesos de cocción rápida, Bol. Soc. Esp. Ceram. V., 21, 1, 15-22 (1982).

11. J.D. Martín-Martín, T. Sanfeliu, D. Gómez-Gras, C. de la Fuente, Mineralogía de las lutitas permotriásicas de la sierra de Vilafamés (Castellón). Aplicación en la industria cerámica, pp. 205-213 en Integración ciencia-tecnología de las arcillas en el contexto tecnológico-social del nuevo milenio. J. Pascual (coord.). Sociedad Española de Arcillas (SEA) 2000.

12. E. Roca, J. Guimerà, R. Salas, Mesozoic extensional tectonics in the southeast Iberian Chain, Geol. Mag. 131, 155-168 (1994).

13. V. Sos, T. Sanfeliu, La geología de la provincia de Castellón y su bibliografía, Sociedad Castellonense de Cultura, Castellón (España) 1983.

14. A. Arche, J. López-Gómez, Una nueva hipótesis sobre las primeras etapas de la evolución tectonosedimentaria de la cuenca pérmico-triásica del SE de la Cordillera Ibérica, Cuad. Geol. Ibérica 16, 115-143 (1992).

15. D. Gómez-Gras, El Permotrías de las Baleares y de la vertiente mediterránea de la Cordillera Ibérica y del Maestrat: Facies y Petrología Sedimentaria (Parte II), Bol. Geol. Min. 104, 5, 467-515 (1993).

16. IGME, Mapa Geológico de España. E 1/200.000. Hoja n ${ }^{\circ} 48$ (Vinaròs), Servicio de Publicaciones del Ministerio de Industria y Energía. Madrid (España) 1985.
17. M. Thiry, N. Forette, J.M. Schmitt. Pp. 51 en Techniques de diffraction des rayons $\mathrm{X}$ et interprétation des diagrammes (Notes Techniques). Centre de Géologie Générale et Minière (ENSMP), Fontainebleau (Francia) 1983.

18. V. Bagán, Características técnicas de las arcillas rojas utilizadas para la fabricación de pavimentos y revestimientos cerámicos, 111-117 en I Encuentro entre la industria de pavimentos y revestimientos cerámicos y la minería de arcillas y caolines en la Comunidad Valenciana (Ponencias). Generalitat Valenciana, Consellería d'Indústria, Comerç i Turisme, Valencia (España), 1988.

19. M. Ruiz-Cruz, M.A. Caballero, Características genéticas y distribución de los minerales de la fracción fina del Trias de la Cordillera Ibérica. I) Caolinita y Dickita, Bol. Geol. Min. LXXXVII, I, 47-56 (1976).

20. J.D. Martín-Martín, D. Gómez-Gras, T. Sanfeliu, Origen de la asociación de minerales de la arcillas del permo-Triásico del Desert de les Palmes (Castellón). Relación con la alteración del zócalo hercínico, Geotemas 8 201-204 (2005).

21. M. Ruiz-Cruz, M.A. Caballero, Características genéticas y distribución de los minerales de la fracción fina del Trias de la Cordillera Ibérica. II) Illita y Mica». Bol. Geol. Min. LXXXVII, II, 177-184 (1976).

22. M. Ruiz-Cruz, M.A. Caballero, Características genéticas y distribución de los minerales de la fracción fina del Trias de la Cordillera Ibérica. II) Clorita, Montmorillonita y Vermiculita, Bol. Geol. Min. LXXXVII, III, 284291 (1976).

23. R. Marfil; M.G. Bonhome; J.A. De la Peña; R. Penha dos Santos; I. Sell., La edad de las illitas en areniscas pérmicas y triásicas de la Cordillera Ibérica mediante el método K/Ar: Implicaciones en la historia diagenética y evolución de la cuenca, Cuad. Geol. Ibérica 20 61-83 (1996).

Recibido: 27.12 .05

Aceptado: 06.03 .06 